\title{
Clinical Research Redirection and Optimization During a Pandemic
}

\author{
Ludmilla Candido Santos, $\mathrm{MD}^{\mathrm{a}}$, Ying Hui Low, MD \\ Konstantin Inozemtsev, $\mathrm{MD}^{\mathrm{c}}$, Alexander Nagrebetsky, MD, Msc ${ }^{\mathrm{b} \text {, * }}$
}

\section{KEYWORDS}

- Pandemic • COVID-19 • Research limitations • Optimization • Publication surge

\section{KEY POINTS}

- Pandemics create acute strains on research resources.

- Research challenges presented by past/current pandemics are similar in nature.

- High demand for information in the setting of a pandemic may decrease the average quality of scientific publications.

- We discuss recommendations for future research, during this public health crisis and others.

\section{INTRODUCTION AND HISTORY}

The first diagnosis in the United States of severe acute respiratory syndrome coronavirus 2 (SARS-CoV-2), also known as coronavirus disease (COVID-19) (which is how this virus is referred to henceforth), was received on January 20, 2020. ${ }^{1,2}$ On March 18, 2020, the United States Food and Drug Administration (FDA) issued an updated guideline for the conduct of clinical trials, which highlighted the need to adhere to social distancing and quarantine guidelines. ${ }^{3}$ Most research activities in major institutions were suspended soon after that, despite the need for scientific research to address the pandemic.

Past pandemics, such as the severe acute respiratory syndrome coronavirus (SARs-CoV-1) in 2002; H1N1 influenza in 2009; Middle East respiratory syndrome, which started in 2012 and is still lingering; and Ebola virus in 2019, have seen a deficient response in clinical research. ${ }^{4}$ Inadequate research collaboration and funding,

L.C. Santos and Y.H. Low contributed equally to the work.

${ }^{a}$ Emergency Medicine Network, Massachusetts General Hospital, Harvard Medical School, 55 Fruit Street, Boston, MA 02114, USA; ${ }^{b}$ Department of Anesthesia, Critical Care and Pain Medicine, Massachusetts General Hospital, Harvard Medical School, 55 Fruit Street, Boston, MA 02114, USA; ' Department of Anesthesiology, Dartmouth-Hitchcock Medical Center, One Medical Center Drive, Lebanon, NH 03756, USA

* Corresponding author.

E-mail address: anagrebetsky@mgh.harvard.edu 
particularly in vulnerable regions, have been identified in a previous review. ${ }^{5}$ In its contemplation of lessons learned from the historical inquiry of pandemic mitigation strategies, the Institute of Medicine ${ }^{6}$ observes that progress in clinical research, particularly with surveillance, rapid communications, modern computing, and epidemic modeling, has the potential to not only mitigate but prevent future pandemics.

The goal of this article is to explore and summarize the effects of pandemics on clinical research and to explore approaches to effectively redirect and optimize clinical research in future pandemics. The discussion is limited to observational and interventional studies in clinical research; basic science research is beyond the scope of this article.

\section{DISCUSSION}

\section{Effects of Pandemics on Components of Clinical Research}

The components of any modern research framework are familiar to all scientists and can be classified broadly into the following steps: (1) generating a research hypothesis and developing a study protocol; (2) obtaining regulatory approval; (3) studying implementation, including securing adequate funding for completion of all study processes, such as interventions and data collection; and (4) analysis and publication of data in the form of a article. ${ }^{7,8}$ Public health emergencies of a new pandemic create acute strains in resources that often affect critical elements of executing a research protocol. These are discussed and summarized in Table 1.

\section{Study design}

Leading up to the appearance of COVID-19, other infectious disease pandemics and large-scale public health emergencies have been cited as events that highlight the need for a coordinated research response. ${ }^{9}$ For instance, the $2009 \mathrm{H} 1 \mathrm{~N} 1$ pandemic, 2010 Haiti earthquake, Deepwater Horizon oil spill, and 2011 Fukushima Daiichi nuclear disaster were highlighted as recent events where the acute strains placed on government agencies, including health care and scientific organizations, limited the ability to address key components of research. ${ }^{9}$ The authors recommend the assembly of a ready team of experts who can generate relevant research questions and prioritize and monitor research needs. ${ }^{9}$ This forms the first step of ensuring conditions for rapid data collection and appropriate human subjects review, mechanisms for rapid funding, and exposure monitoring. ${ }^{9}$

It also is paramount to ensure that research planning is carried out in an ethical manner despite the emerging disaster, by engaging both public and scientific experts to address specific needs of the different communities involved in research. These discussions should address ethical questions that arise during public health emergencies and the risk that research might be perceived as an exploitation of vulnerable people or communities in a state of disaster. ${ }^{9}$ Investigators also have underscored the importance of abiding by systematic risk and ethical evaluations when assessing the ethics of pandemic-related research, particularly in high-risk clinical trials, such as earlyphase trials of potential vaccines for COVID-19. ${ }^{10}$ Such safety considerations has to be balanced deftly with the need for quicker regulatory approval.

\section{Regulatory approval}

Even before the current pandemic, investigators have called for the development of more precise consensus guidelines addressing the waiver of informed consent in emergency research. ${ }^{11}$ In 2020, the challenges of carrying out emergency research with exceptions for informed consent were highlighted, with some investigators calling 


\begin{tabular}{|c|c|}
\hline $\begin{array}{l}\text { Steps in the Research } \\
\text { Process }\end{array}$ & Effects in the Setting of a Pandemic \\
\hline \multicolumn{2}{|l|}{ Study design } \\
\hline Generating a hypothesis & $\begin{array}{l}\text { Lack of expertise in emerging disease } \\
\text { Limited expert availability with clinician-scientists } \\
\text { responding to high demand in clinical care }\end{array}$ \\
\hline Study protocol design & $\begin{array}{l}\text { Prioritization of studies that yield quick results rather } \\
\text { than long-term outcomes } \\
\text { Delays in gathering preliminary data that may help } \\
\text { inform main study design (eg, for a power analysis) } \\
\text { Difficulty ensuring proper risk and ethics analysis and } \\
\text { that research planning is ethical and not seen as an } \\
\text { exploitation of vulnerable communities }\end{array}$ \\
\hline \multicolumn{2}{|l|}{ Regulatory approval } \\
\hline Institutional board review & $\begin{array}{l}\text { Potential for delay with institutional board review and } \\
\text { approval }\end{array}$ \\
\hline FDA approval & $\begin{array}{l}\text { Potential fast-tracking for COVID-19 studies } \\
\text { Potential delay for non-COVID-19 studies }\end{array}$ \\
\hline \multicolumn{2}{|l|}{ Study implementation } \\
\hline Funding & $\begin{array}{l}\text { Allocation of funding toward more immediate health } \\
\text { care needs }\end{array}$ \\
\hline $\begin{array}{l}\text { Intervention delivery } \\
\text { and data collection }\end{array}$ & $\begin{array}{l}\text { Site closures } \\
\text { Travel limitations } \\
\text { Interruptions to supply chain } \\
\text { Limited access to research facilities and equipment due } \\
\quad \text { to official stay-at-home orders } \\
\text { Limited access to the delivery of interventions } \\
\text { Disrupted patient screening and recruitment } \\
\text { Limited in-person baseline and follow-up data } \\
\text { collection }\end{array}$ \\
\hline \multicolumn{2}{|l|}{ Data review and publication } \\
\hline Data analysis & $\begin{array}{l}\text { Redirection of resources to pandemic-related projects } \\
\text { Disrupted work environment and team dynamics due to } \\
\text { remote work settings }\end{array}$ \\
\hline Article writing and review & $\begin{array}{l}\text { Perceived pressure to produce results } \\
\text { Decreased peer review standards due to high demand } \\
\text { for information } \\
\text { Potential impact on quality of publications }\end{array}$ \\
\hline
\end{tabular}

for the need to offer appropriate means for rapid consultation to support such exceptions. $^{12}$

In addition, investigators observed the need for greater regulatory flexibility during pandemics to manage both the goals of protecting participants and promoting the development of high-quality evidence that informs patient care during the pandemic. $^{12}$ Although many institutional review boards (IRBs) also took steps to speed the review of COVID-19 protocols, this led to a challenge of heightened workload and personal responsibilities for IRB members, particularly at academic institutions, and may not be sustainable. ${ }^{12}$

Furthermore, the traditional safeguards for research that involves incarcerated persons, which was a population of patients heavily impacted by COVID-19, possibly may 
be to their detriment, and investigators have suggested the need to allow such research when there is the prospect of direct benefit to these individuals in custody. ${ }^{12}$

During COVID-19, the FDA also helped conduct ultrarapid protocol reviews for research subject to investigational new drug and investigational device exemption requirements, as part of the Coronavirus Treatment Acceleration Program. ${ }^{13}$

\section{Study implementation}

The inaccessibility of trial participants and research personnel due to social distancing rules during COVID-19 led to delays in patient enrollment, and operational gaps in clinical trials may have a negative impact on data integrity. ${ }^{14}$

Patient screening and recruitment. The success and generalizability of clinical trials depend considerably on extensive participant enrollment. ${ }^{15}$ During COVID-19, patient recruitment and ease of conducting in-person visits were significantly affected, not only by official policy limiting nonessential movement but also by fears of a poorly characterized disease among participants and caregivers. ${ }^{15,16}$

Consent process. With isolation or physical distancing requirements, the FDA recommended the use of electronic consent via the COVID MyStudies mobile device application or the use of phone or videoconferencing. A comprehensive discussion with a potential study participant, however, is time consuming - for instance, the discussion of every possible alternative to enrollment in a given COVID-19 protocol, particularly in the early phases of a pandemic, when available alternatives were rapidly changing. It has been suggested that regulations be revised to allow more flexibility for only context-appropriate disclosures to patients. ${ }^{12}$

Intervention delivery and data collection. COVID-19 resulted in extensive travel restrictions and site closures, in addition to the diversion of nonessential hospital space, including research areas, to enhance patient isolation. ${ }^{17}$ Investigators also have to consider the relative risks and benefits of conducting research activities and provide a safe environment and, where appropriate, reassurance to participants. ${ }^{16}$ In addition, disruption of supply chains for investigational drugs further jeopardized delivery of study interventions. ${ }^{17}$ Protocols often required modification to ensure adherence to intervention and measurement of outcomes, including remote data collection by telephone, video or telehealth platforms, and carrying out follow-up testing at home where possible. ${ }^{18,19}$ Other than transitioning to remote operations, where possible, the pandemic experience also has highlighted the value of research networks - established pathways for sharing information between sites can help speed up the process of gathering valuable data and may overcome obstacles, such as those encountered during the current pandemic. ${ }^{19}$

\section{Data review and publication}

A step in the research process that may seem unhindered by the COVID-19 pandemic is the publication of articles, although it remains debatable whether this is a marker of research success. It may be contended that even prior to the pandemic, publication was a process carried out entirely remotely, so it was least affected by the new pandemic rules.

This apparent success in COVID-19 research, however, is marred by the following criticisms. First, the number of publications also appears to be heavily inflated by nonpeer-reviewed or nonoriginal research, such as editorials and opinion articles, many of which are cited later. Second, the publication and visibility of potentially impactful non-COVID-19 articles may be affected by the overwhelming demand for COVID19 research. 


\section{Publication Surge}

As illustrated in Fig. 1, the number of publications on COVID-19 was unparalleled. For comparison, after 6 months of the report of the first case of SARS-CoV-1 in February $2003,{ }^{20} 929$ articles had been published as situation unfolded; and by the sixth month after the 2009 pandemic of H1N1 Influenza started, 1245 articles about the virus were available on PubMed-indexed journals. By contrast, in June 2020, the number of articles published on COVID-19 was approximately 30 -times higher $(35,891)$ than in the previous pandemics. ${ }^{21-23}$

The average quality of early COVID-19 publications, however, was met with harsh critique among the both scientific and journalism communities, for the peer review step frequently was skipped in a fast-tracking publication process. ${ }^{21-23}$ This pandemic also saw many investigators using preprint servers to disseminate their work. ${ }^{24}$ These servers, such as medRxiv, sponsored by Yale University, have become popular sources of information among journalists. Some journals, as The Lancet, also make preprints available to the public, and, despite containing a disclaimer that these are articles that have not completed the peer review process or been accepted, ${ }^{25}$ they have been used as references by journalists who are looking for the latest updates on COVID-19. ${ }^{26,27}$ Multiple preprints on COVID-19 that have been covered by popular media on COVID-19 have been written and retracted within the same year. ${ }^{28}$

Although preprints and articles on preliminary results are much criticized, there are arguments in favor of their utility. Early publication of research methodology and findings allows for earlier detection of methodological issues by the readers. Publication of preliminary results may allow younger, less established researchers to disseminate their ideas and data without having to subject themselves to prohibitive criticism from reviewers of well-known journals, while still receiving credits for their efforts.

These potential benefits, however, must be weighed against the risk of low-quality, non-peer-reviewed research fueling media-driven panic or resulting in inappropriate clinical and policy decisions based on erroneous data, which both could cause harm to individuals and compromise scientific integrity.

Even articles published in reputable journals have been retracted, including from Annals of Internal Medicine, ${ }^{33}$ The Lancet, ${ }^{34,35}$ and The New England Journal of Medicine. ${ }^{36}$ A recent review reports that, despite the large number of publications, only a fraction of the published studies fulfilled the principles of evidence-based practice. ${ }^{37}$

The publication onslaught also may reflect a prioritization of studies that yield quick results rather than long-term outcomes. Other investigators also have called for

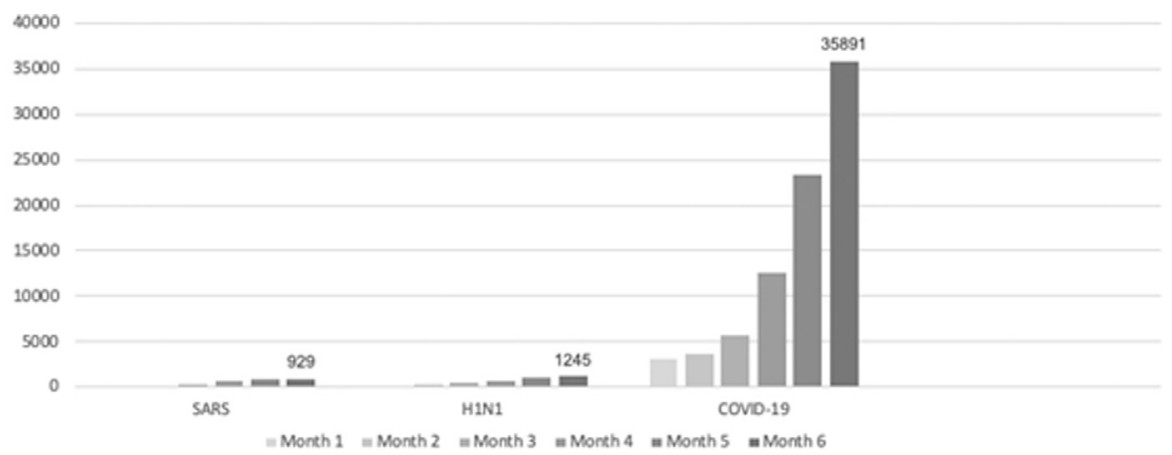

Fig. 1. Cumulative number of publications per month on PubMed during SARS-CoV-1, H1N1, and COVID-19 outbreaks as of August 28, 2020. 
researchers to collaborate in larger high-quality investigations, rather than duplicate research in multiple small trials with analogous hypotheses, which may be easier to carry out but less likely to yield precise results. ${ }^{32}$

\section{Concurrent Non-COVID-19 Research}

As discussed previously, a veritable explosion of COVID-19 related trials, now numbering in the thousands, represents a significant shift in scientific effort and energy expenditure. ${ }^{38}$ Other key basic and clinical research endeavors have been stymied or halted by mandatory facility shutdowns, staff quarantine and distancing measures, suspension of animal and human clinical trials, and loss or reallocation of funding. ${ }^{39}$

In addition to serving as a significant setback for scientific innovation itself, this has had a negative impact on several patients without COVID-19, including many in vulnerable populations, such as those receiving cancer care and immunosuppressive therapy. ${ }^{40}$ Many were participating or expecting enrollment in clinical trials, a significant proportion of which was suspended or halted altogether ${ }^{41}$ or had recruitment drop precipitously. ${ }^{42}$

Withdrawal of funding from critical research should be avoided or limited whenever possible due to its long-term ramifications for both affected patients, and the scientific community at large. Importantly, ongoing work in related fields may elucidate underlying mechanisms of pathogenesis and treatment of COVID-19 itself, and vice versa. ${ }^{43}$

\section{Research Redirection and Collaboration}

With a bevy of researchers addressing the topic from multiple angles, there also has been a growing concern over studies being inadequately powered and suffering from redundance and heterogeneity in methodology and outcome measurement. As such, there is a need for coordination and establishment of clear guidelines for research and reporting. ${ }^{44}$

Several international consortia, including diverse specialty groups ${ }^{45}$ and global health organizations, ${ }^{46}$ have offered core outcome sets for trials pertinent to COVID-19. At minimum, these core outcomes should be addressed to maximize data utility and facilitate international collaboration and data pooling efforts.

To encourage international collaboration further, a group of National Science and Technology Advisors spanning numerous countries issued a call to publishers to make COVID-19 publications and associated data freely available in the public domain, ${ }^{47}$ highlighting the importance of information and data sharing as means of increasing scientific yield and building more robust data sets for current and future clinical trials. ${ }^{48}$

Furthermore, as research efforts begin bearing fruit, several important differences in symptomatology, complications, and burden of disease within and between populations have emerged. Following the initial paucity of information, geographic, genetic, gender-based, racial, cultural, and socioeconomic variations, among others, have demonstrated important and sometimes drastic differences with significant implications for both treatment and preventative measures. ${ }^{49}$

\section{Research Optimization}

As more data emerge, attention must shift from simply examining comparative epidemiology to utilizing it to guide further research needs, address contributing inequities, and help protect those groups and populations that emerge as most vulnerable. ${ }^{50} \mathrm{At}$ the same time, carefully controlling for a maximal number of such variables during subgroup analysis serves to eliminate spurious correlations and maximize generalizability, crucial to both informed clinical decisions and policy making. ${ }^{51}$ 
Some of the obstacles posed by the pandemic also can be overcome by enhancing collaborative efforts between researchers, for instance, in the form of data registries. National societies frequently have the capacity to facilitate and coordinate research efforts. For example, the American Society of Anesthesiologists Committee on Critical Care Medicine has served as a platform for networking and site recruitment for the COVID-19 ICU Registry. The COVID-19 ICU Registry ${ }^{52}$ is a multi-institutional and international collaboration to collect critical care and respiratory data about patients with COVID-19, with the goals of being able to identify key risk factors for severe illness and disease patterns and potentially to assess treatment efficacy. The CORAL series of studies ${ }^{53}$ is another example of a multicenter effort to collect and share data between tens of institutions nationwide.

Health care workers themselves appear to be at elevated risk for contracting the disease, and the intubateCOVID registry, which was designed to track various aspects and outcomes of airway management of infected patients, demonstrated a notable incidence of COVID-19 symptoms, confirmed infection, and sequelae affecting approximately $10 \%$ of intubating staff. ${ }^{54}$ This, compounded with health care workers' increased risk of being asymptomatic or presymptomatic carriers ${ }^{55}$ capable of transmitting the disease to their families and others, ${ }^{56}$ can significantly affect both productivity and attitudes toward remaining clinically and academically active.

\section{SUMMARY}

The COVID-19 pandemic has seen many hurdles to crucial research processes, in particular those that depend on personnel interactions, in providing safeguards against the incipient infectious disease. At the same time, there was a rapid redirection of research, driven by popular and social media and demand for pandemic-related content, to the detriment of non-COVID-19 research and perhaps to COVID-19 research itself.

This pandemic has provided critical lessons to the authors, who believe the following will be essential for future research success: (1) regional, national, and global scientific societies need to rapidly identify strategic high-yield areas for research based on preliminary data and coordinate efforts at acquiring reliable quality data from multiple sources for collaborative data analysis, including in the form of data registries; (2) prompt recognition by regulatory authorities of areas of flexibility that are relevant to the new crisis; and (3) publishers should lay out guidelines for what constitutes a significant investigation and help provide a constant source of quality control for the research enthusiasm that comes with a novel research question.

Although there is little motivation to create and update contingency strategies for research coalitions, regulation, and publishing during periods of nonemergency, these efforts are likely to be most successful when preplanned and pretested at a time of relative abundance of resources. It would be prudent for the scientific community to maintain processes learned during COVID-19 so that these lessons do not need to be relearned during the next public health crisis.

\section{ACKNOWLEDGMENTS (FUNDING INFORMATION)}

This study is funded by T32 NIH grant: 2T32GM007592-41.

\section{DISCLOSURE}

The authors have no conflicts of interest to disclose. 


\section{REFERENCES}

1. Holshue ML, DeBolt C, Lindquist S, et al. First Case of 2019 Novel Coronavirus in the United States. N Engl J Med 2020;382(10):929-36.

2. Harcourt J, Tamin A, Lu Xea. Severe acute respiratory syndrome coronavirus 2 from patient with coronavirus disease, United States. 2020. Available at: https:// wwwnc.cdc.gov/eid/article/26/6/20-0516_article. Accessed October 27, 2020.

3. U.S. Food and Drug Administration. Clinical Trial Conduct During the COVID-19 Pandemic. 2020. Available at: https://www.fda.gov/drugs/coronavirus-covid-19drugs/clinical-trial-conduct-during-covid-19-pandemic. Accessed October 27, 2020.

4. Rojek AM, Horby PW. Modernising epidemic science: enabling patient-centred research during epidemics. BMC Med 2016;14(1):212.

5. Sigfrid L, Maskell K, Bannister PG, et al. Addressing challenges for clinical research responses to emerging epidemics and pandemics: a scoping review. BMC Med 2020;18(1):190.

6. Institute of Medicine (US). Ethical and Legal Considerations in mitigating pandemic disease: Workshop summary. Washington, DC: The National Academies Press; 2007.

7. Thiese MS. Observational and interventional study design types; an overview. Biochem Med (Zagreb) 2014;24(2):199-210.

8. Eriksen MB, Frandsen TF. The impact of patient, intervention, comparison, outcome (PICO) as a search strategy tool on literature search quality: a systematic review. J Med Libr Assoc 2018;106(4):420-31.

9. Lurie N, Manolio T, Patterson AP, et al. Research as a part of public health emergency response. N Engl J Med 2013;368(13):1251-5.

10. Bull S, Jamrozik E, Binik A, et al. SARS-CoV-2 challenge studies: ethics and risk minimisation [published online ahead of print, 2020 Sep 25]. J Med Ethics 2020. https://doi.org/10.1136/medethics-2020-106504. medethics-2020-106504.

11. Vaslef SN, Cairns CB, Falletta JM. Ethical and regulatory challenges associated with the exception from informed consent requirements for emergency research: from experimental design to institutional review board approval. Arch Surg 2006; 141(10): 1019-23 [discussion: 1024].

12. Fernandez Lynch H, Dickert NW, Zettler PJ, et al. Regulatory flexibility for COVID19 research. J L Biosci 2020;7(1):Isaa057.

13. U.S. Food and Drug Administration. Coronavirus Treatment Acceleration Program (CTAP). 2020. Available at: https://www.fda.gov/drugs/coronavirus-covid-19drugs/coronavirus-treatment-acceleration-program-ctap. Accessed October 27, 2020.

14. Sathian B, Asim M, Banerjee I, et al. Impact of COVID-19 on clinical trials and clinical research: A systematic review. Nepal J Epidemiol 2020;10(3):878-87.

15. Vidoni ED, Szabo-Reed A, Kang C, et al. The IGNITE trial: Participant recruitment lessons prior to SARS-CoV-2. Contemp Clin Trials Commun 2020;20:100666.

16. Padala PR, Jendro AM, Padala KP. Conducting Clinical Research During the COVID-19 pandemic: investigator and participant perspectives. JMIR Public Health Surveill 2020;6(2):e18887.

17. Davis S, Pai S. Challenges and opportunities for sponsors in conducting clinical trials during a pandemic. Perspect Clin Res 2020;11(3):115-20.

18. McDermott MM, Newman AB. Preserving clinical trial integrity during the coronavirus pandemic. JAMA 2020;323(21):2135-6. 
19. van Koningsbruggen-Rietschel S, Dunlevy F, Bulteel V, et al. SARS-CoV-2 disrupts clinical research: the role of a rare disease-specific trial network. Eur Respir J 2020;56(3).

20. Cherry JD. The chronology of the 2002-2003 SARS mini pandemic. Paediatr Respir Rev 2004;5(4):262-9.

21. Teixeira da Silva JA, Tsigaris P, Erfanmanesh M. Publishing volumes in major databases related to Covid-19. Scientometrics 2020;1-12. https://doi.org/10.1007/ s11192-020-03675-3.

22. Nowakowska J, Sobocinska J, Lewicki M, et al. When science goes viral: The research response during three months of the COVID-19 outbreak. Biomed Pharmacother 2020;129:110451.

23. Fidahic M, Nujic D, Runjic R, et al. Research methodology and characteristics of journal articles with original data, preprint articles and registered clinical trial protocols about COVID-19. BMC Med Res Methodol 2020;20(1):161.

24. Krumholz HM. The End of Journals. Circ Cardiovasc Qual Outcomes 2015;8(6): 533-4.

25. Preprints with The Lancet. Available at: https://www.ssrn.com/index.cfm/en/thelancet/. Accessed October 27, 2020.

26. Flier JS. Covid-19 is reshaping the world of bioscience publishing. First Opinion Web site. 2020. Available at: https://www.statnews.com/2020/03/23/biosciencepublishing-reshaped-covid-19/. Accessed October 27, 2020.

27. Packer M. Does Peer Review Still Matter in the Era of COVID-19?. 2020. Available at: https://www.medpagetoday.com/blogs/revolutionandrevelation/86465. Accessed 16 Oct 2020.

28. Retracted coronavirus (COVID-19) papers. 2020. Available at: https:// retractionwatch.com/retracted-coronavirus-covid-19-papers/. Accessed October 27, 2020.

29. Dinis-Oliveira RJ. COVID-19 research: pandemic versus "paperdemic", integrity, values and risks of the "speed science. Forensic Sci Res 2020;5(2):174-87.

30. Ioannidis JPA. Coronavirus disease 2019: the harms of exaggerated information and non-evidence-based measures. Eur J Clin Invest 2020;e13223.

31. Balaphas A, Gkoufa K, Daly MJ, et al. Flattening the curve of new publications on COVID-19. J Epidemiol Community Health 2020;74(9):766-7.

32. London AJ, Kimmelman J. Against pandemic research exceptionalism. Science 2020;368(6490):476-7.

33. Bae S, Kim MC, Kim JY, et al. Effectiveness of Surgical and Cotton Masks in Blocking SARS-CoV-2: A Controlled Comparison in 4 Patients. Ann Intern Med 2020;173(1):W22-3.

34. Mehra MR, Desai SS, Ruschitzka F, et al. RETRACTED: Hydroxychloroquine or chloroquine with or without a macrolide for treatment of COVID-19: a multinational registry analysis. Lancet 2020. https://doi.org/10.1016/S0140-6736(20)31180-6.

35. Funck-Brentano C, Salem JE. Chloroquine or hydroxychloroquine for COVID-19: why might they be hazardous? Lancet 2020. https://doi.org/10.1016/S01406736(20)31174-0.

36. Mehra MR, Desai SS, Kuy S, et al. Cardiovascular Disease, Drug Therapy, and Mortality in Covid-19. N Engl J Med 2020;382(25):e102.

37. Lv M, Luo X, Estill J, et al. Coronavirus disease (COVID-19): a scoping review. Euro Surveill 2020;25(15).

38. US National Library of Medicine. Available at: https://clinicaltrials.gov. Accessed October 26, 2020. 
39. Keswani SG, Parikh UM, Gosain A, et al. Impact of the coronavirus disease 2019 pandemic on surgical research and lessons for the future. Surgery 2020;169(2): 257-63 [published online ahead of print, 2020 Sep 19].

40. Sharpless NE. COVID-19 and cancer. Science 2020;368(6497):1290.

41. Rosenbaum L. The Untold Toll - The Pandemic's Effects on Patients without Covid-19. N Engl J Med 2020;382(24):2368-71.

42. Bailey C, Black JRM, Swanton C. Cancer research: the lessons to Learn from COVID-19. Cancer Discov 2020;10(9):1263-6.

43. Auletta JJ, Adamson PC, Agin JE, et al. Pediatric cancer research: Surviving COVID-19. Pediatr Blood Cancer 2020;67(9):e28435.

44. Bauchner H, Fontanarosa PB. Randomized Clinical Trials and COVID-19: Managing Expectations. JAMA 2020;323(22):2262-3.

45. Tong A, Elliott JH, Azevedo LC, et al. Core Outcomes Set for Trials in People With Coronavirus Disease 2019. Crit Care Med 2020;48(11):1622-35.

46. Marshall JC, Murthy S, Diaz J, et al. WHO Working Group on the Clinical Characterisation and Management of COVID-19 infection. A minimal common outcome measure set for COVID-19 clinical research. Lancet Infect Dis 2020;20(8):e192-7 [published correction appears in Lancet Infect Dis 2020;20(10):e250].

47. WhiteHouse.gov. Open Acces Letter from CSAs. 2020. Available at: https://www. whitehouse.gov/wp-content/uploads/2020/03/COVID19-Open-Access-Letter-fromCSAs. Equivalents-Final.pdf. Accessed October 28, 2020.

48. Navar AM, Pencina MJ, Rymer JA, et al. Use of Open Access Platforms for Clinical Trial Data. JAMA 2016;315(12):1283-4.

49. Price-Haywood EG, Burton J, Fort D, et al. Hospitalization and mortality among black patients and white patients with covid-19. N Engl J Med 2020;382(26): 2534-43.

50. Laurencin CT, McClinton A. The COVID-19 Pandemic: a Call to Action to Identify and Address Racial and Ethnic Disparities. J Racial Ethn Health Disparities 2020; 7(3):398-402.

51. Kabarriti R, Brodin NP, Maron MI, et al. Association of Race and Ethnicity With Comorbidities and Survival Among Patients With COVID-19 at an Urban Medical Center in New York. JAMA Netw Open 2020;3(9):e2019795.

52. Massachusetts General Hospital. COVID-19 ICU REGISTRY: A Multicenter International Data Repository. Available at: https://www.massgeneral.org/anesthesia/ research/covid-19-icu-registry. Accessed October 31, 2020.

53. RED CORAL: PETAL Repository of Electronic Data COVID-19 Observational Study. Available at: https://petalnet.org/studies/public/redcoral. Accessed October 31, 2020.

54. El-Boghdadly K, Wong DJN, Owen R, et al. Risks to healthcare workers following tracheal intubation of patients with COVID-19: a prospective international multicentre cohort study. Anaesthesia 2020;75(11):1437-47.

55. Stock AD, Bader ER, Cezayirli P, et al. COVID-19 Infection Among Healthcare Workers: Serological Findings Supporting Routine Testing. Front Med (Lausanne) 2020;7:471.

56. Buitrago-Garcia D, Egli-Gany D, Counotte MJ, et al. Occurrence and transmission potential of asymptomatic and presymptomatic SARS-CoV-2 infections: A living systematic review and meta-analysis. PLoS Med 2020;17(9):e1003346. 